\title{
An analysis of ocular features and vision loss at presentation and after treatment in systemic lupus erythematosus in 60 patients
}

\author{
Sruthi MS', Annamalai $\mathbf{R}^{2}$, Muthukumar $\mathbf{M}^{3}$ \\ ${ }^{1}$ Dr. M. Shantha Sruthi, Postgraduate in Ophthalmology, ${ }^{2}$ Dr. Radha Annamalai Associate Professor of Ophthalmology, \\ ${ }^{3}$ Prof. Muthayya Muthukumar, Senior Consultant in Ophthalmology. All are affiliated with Sri Ramachandra University, \\ Chennai, India.
}

Address for correspondence: Dr. Radha Annamalai, Email: drradhaannamalai@yahoo.co.in

\begin{abstract}
Introduction: Systemic lupus erythematosus (SLE) is a chronic, inflammatory, multisystem autoimmune disease and ocular involvement occurs in several forms. It may be the first indicator of underlying SLE. Early identification and precise treatment of the disease can reduce blindness. The objective of this study was to analyze the causes of visual loss before and during treatment. Methods: This is a retrospective cohort study on 60 patients over 2 years in multispecialty, tertiary eye care hospital. Ophthalmic examination and routine and ancillary investigations were performed. Patients were followed up for at least one year to assess the progress of ocular features, their response to treatment and development of complications. Case records from a referral practice in patients with systemic lupus erythematosus, with diagnosis of dry eye, peripheral ulcerative keratitis, episcleritis, scleritis, uveitis, retinitis or optic neuropathy were analyzed. Results: In our study, the incidence of episcleritis was most frequent followed by dry eye, retinopathy and keratitis. Age group affected was 34 to 52 years and more in women. Scleritis was associated with decreased vision and maximum complications. Following treatment, cataract was the commonest cause of blindness. Conclusion: Our study showed the presence of simultaneous, multiple ocular complications which make treatment and follow up very crucial to avoid blindness. Ocular signs that occurs in SLE can be vision threatening and requires immediate assessment and management by an ophthalmologist. Drugs used to treat SLE may also cause blindness and coordinated treatment between rheumatologist, physician and ophthalmologist needs to be adopted.
\end{abstract}

Key words: Systemic Lupus Erythematosus, Uveitis, Retinitis, Chloroquine, Blindness

\section{Introduction}

Systemic lupus erythematosus (SLE) isa chronic, autoimmune, connective tissue disease with a relapsing and remitting clinical course. Multi organ involvement results from the production and circulation of autoantibodies and immune complexes with high levels of systemic inflammation and end- organ damage. The deposition of these antibodies cause manifestations in multiple systems and the eye is one such organ which can be affected during the active phase of the disease or even following treatment [1]. Ocular inflammation can be the earliest sign of the underlying disease and a diagnosis of this condition the eye would enable earlier and precise diagnosis with treatment intervention. It has been proposed that SLE causes ocular involvement by

Manuscript received $12^{\text {th }}$ April 2016

Reviewed: $22^{\text {nd }}$ April 2016

Author Corrected: $4^{\text {th }}$ May 2016

Accepted for Publication $16^{\text {th }}$ May 2016 several mechanisms including immune complex deposition, antibodies, vasculitis and thrombosis. Depositions of such immune complexes have been identified in the vasculature of the conjunctiva, retina, choroid, sclera, ciliary body and visual pathway [2]. Drugs such as oral corticosteroids, immunosuppressive agents and hydroxychloroquine were used in the treatment of SLE and treatment itself may cause vision loss due to cataract or retinopathy. Anterior segment involvement is seen as keratoconjunctivitis sicca (KCS), conjunctivitis, episcleritis, anterior scleritis, keratitis and iritis. Posterior segment disease causes retinopathy, choroidopathy, posterior scleritis and optic neuropathy. About one third of patients affected by SLE have ocular manifestations of which the most common is KCS and the most vision threatening is retinal vasculitis and optic neuritis. 
The presence of posterior segment involvement is a strong indicator of severe systemic disease and requires immediate liaison between the physician, rheumatologist and ophthalmologist [3]. Patients with ocular features need to be treated both with topical eye drops and local injections for inflammation apart from systemic oral and intravenous drugs. Initial systemic management is in the form of high- dose oral or intravenous corticosteroids with immunosuppressive drugs or biologics. SLE is more common in people belonging to African and Asian race and thrombotic complications are reported to be more frequent in Caucasian population [4]. The median age of presentation is between the third to fifth decades of life and the incidence is nine times more common in women. In this present study that we performed we analyzed the results of ocular examinations in a cohort of 75 eyes of 60 patients with SLE. Our data reinforces the high incidence of ocular manifestations in systemic lupus erythematosus but for the most part emphasizes on the post treatment ocular sequelae in these patients. Hence although the systemic disease is controlled by therapy, the patient continues to require monitoring for the detection of ophthalmic complications.

\section{Materials and Methods}

This study was performed on 60 patients over a period of 2 years to analyze the ocular features and vision loss in systemic lupus erythematosus before, during and after completion of treatment at a tertiary care eye center in India. It was performed in concurrence with the departments of dermatology, rheumatology and internal medicine and was a retrospective cohort study conducted on all new patients with SLE who presented to the ophthalmology outpatient department with visual complaints. Prior to the study, ethics committee clearance was obtained. Inclusion criteria were patients above 18 years of age with SLE and both newly diagnosed and old patients under treatment were enrolled. Patients with prior cataract, glaucoma, and immunosuppression, history of allergy, other autoimmune diseases and systemic co morbidities such as diabetes, hypertension and infectious diseases were excluded from the study. In the first visit, the patients were categorized into two study groups based on whether they were on treatment for SLE or not. Retrospectively, 60 case records from a referral practice with a general diagnosis of dry eye, peripheral ulcerative keratitis, episcleritis, scleritis, uveitis, retinitis, chorioretinitis or optic neuropathy were reviewed. Their medical history, clinical signs, investigations were obtained and specific systemic correlation at diagnoses was performed. Patients had complete ophthalmic and medical examinations.

A complete ophthalmic evaluation was performed on all patients. After obtaining a detailed history, slit lamp examination, indirect ophthalmoscopy, biomicroscopy, tonometry and refraction was done. Ancillary investigations such as fundus fluorescein angiography, optical coherence tomography and B scan ultrasonography were done whenever required. Laboratory workup comprised of complete blood count, erythrocyte sedimentation rate (ESR), rheumatoid factor, antinuclear antibody, VDRL/FTA-ABS, serum uric acid, urinalysis, Mantoux test and chest x-ray. Other investigations used were serum creatinine, urine analysis microscopy and antibodies to double stranded DNA. Patients were followed up for at least one year and during each visit a clinical examination and relevant investigations were performed. During active disease, weekly reviews were performed for one month. After response to treatment or resolution, monthly reviews for one year were done to look for ocular complications, visual improvement and systemic improvement. Blood workup including complete blood count, liver function, renal function and blood sugar was done when treatment with oral steroids $(1 \mathrm{mg} / \mathrm{kg}$ bodyweight/ day) or immunosuppressive drugs were planned.

\section{Results}

An analysis of 60 patients with ocular features in SLE was done for over 2 years and minimum period of follow up of patients was one year. There were $52(60 \%)$ females and $8(40 \%)$ males with an age range of 20 to 60 years and a mean age of 40 (SD +/- 15). Among them, $9 \%$ had corneal complications, 36\% had KCS, $13 \%$ had punctate corneal erosions and $1 \%$ had peripheral corneal ulcer due to the lupus disease activity itself. Episcleritis was seen in $57 \%$ and was the presenting sign of SLE in 9\%. Diffuse epicleritis was noted in $60 \%$ and nodular episcleritis in $4 \%$ of patients. The difference in the pattern of presentation of episcleritis was found to be statistically significant with $\mathrm{p}=0.003$. Scleritis was noted in $1 \%$. It was a case of posterior scleritis and no anterior scleritis occurred in our study cohort. Anterior uveitis was seen in $3 \%$. Retinal involvement was seen in $16 \%$ of our patients. Among those with retinal involvement, vasculitis was seen in $5 \%$. Vasculitis was associated with CNS disease in $3 \%$ of our patients. Other signs of retinopathy were macular 
edemas, hemorrhages and cotton wool spots. Optic disc edema was noted in $2 \%$ of our patients. We also observed in our study, a patient who presented to us with simultaneous multiple ocular manifestations. She was found to have diffuse episcleritis, anterior uveitis, vitritis and optic disc edema. The ocular complications that we noted on patients undergoing treatment were retinal pigment epithelium atrophy and corneal deposits. Our patients were treated with tablet prednisolone $1 \mathrm{mg} / \mathrm{kg}$ / body weight, azathioprine $150 \mathrm{mg}$ daily and tapered to $50 \mathrm{mg}$, hydroxychloroquine $300-400 \mathrm{mg}$ daily. Following treatment, resolution of signs and improvement of vision was noted in $41 \%$. The main causes of defective vision after treatment were cataract in $21 \%$, retinal pigment epithelium atrophy in $7 \%$ and maculopathy in $4 \%$ and optic atrophy in $1 \%$ of patients. The complications of SLE after 1 year of follow up that we observed were dry eye in $48 \%$, progression of cataract in $21 \%$, scleral necrosis in $2 \%$, and vascular occlusion in $1 \%$.

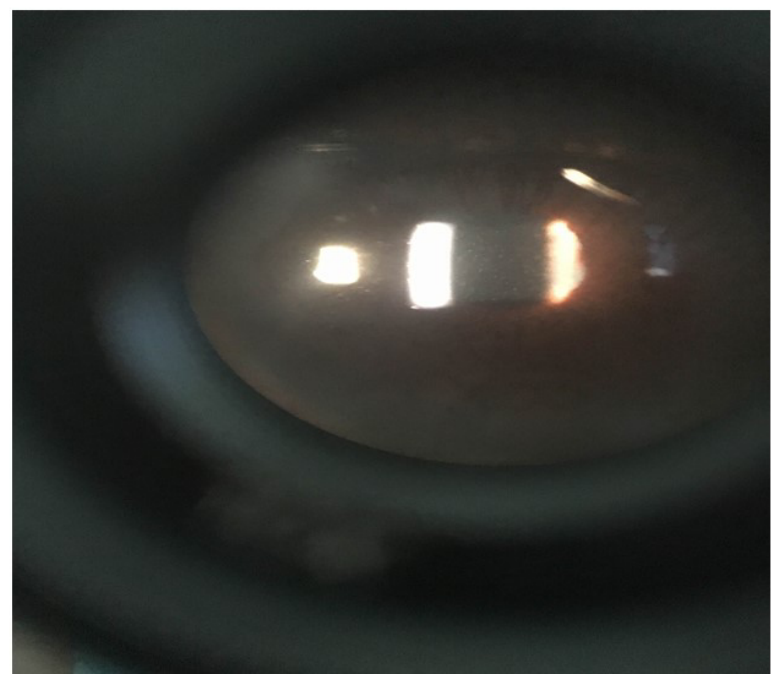

Figure 1: Acute anterior uveitis in SLE.

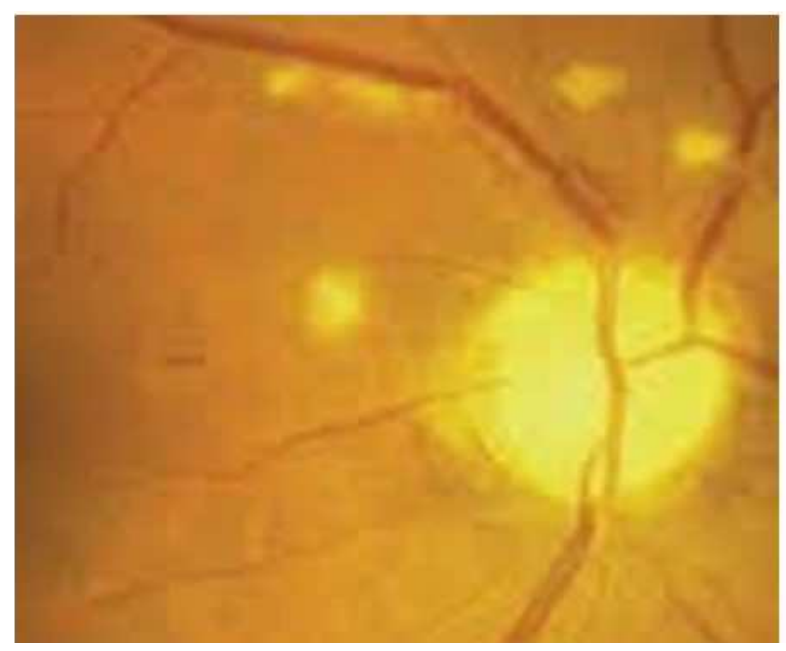

Figure 3: Vasculitis with optic atrophy.

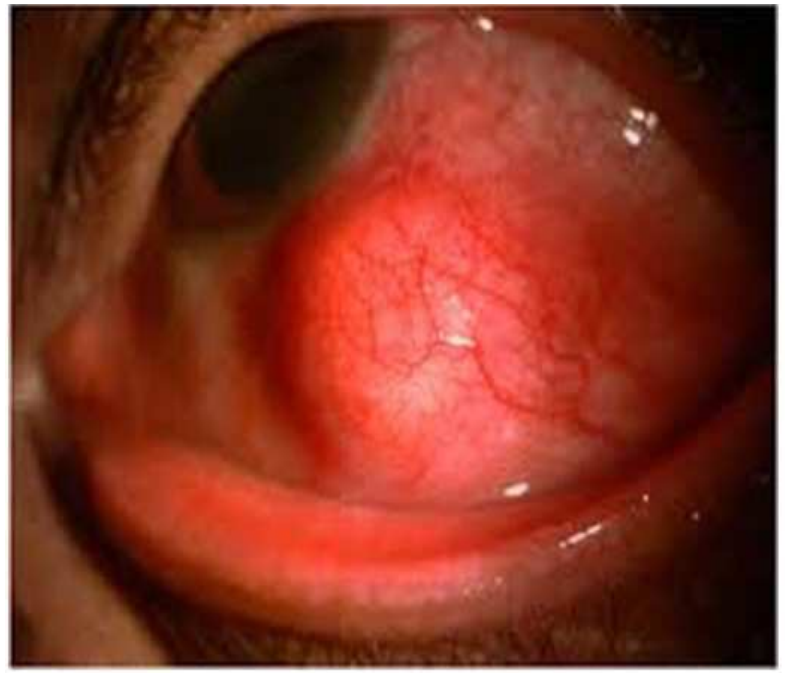

Figure 2: Nodular episcleritis.

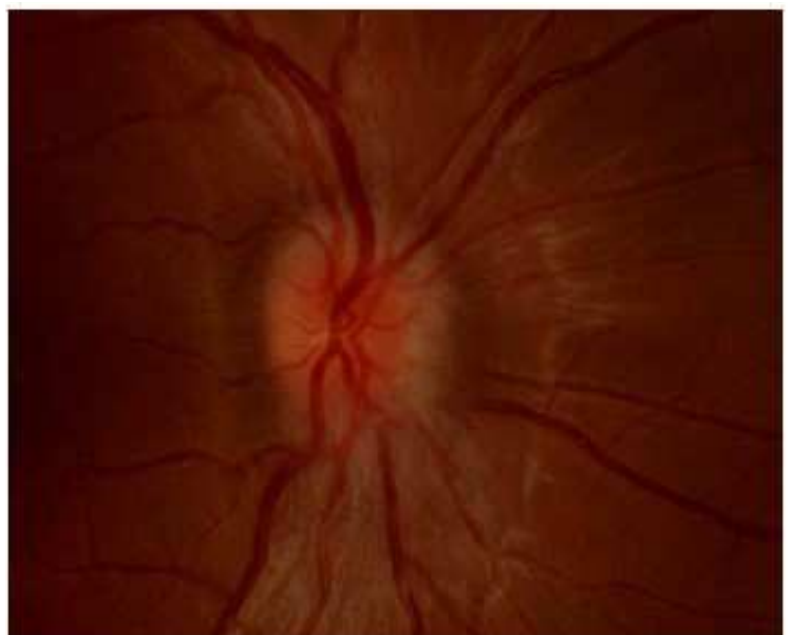

Figure 4: Optic disc oedema. 


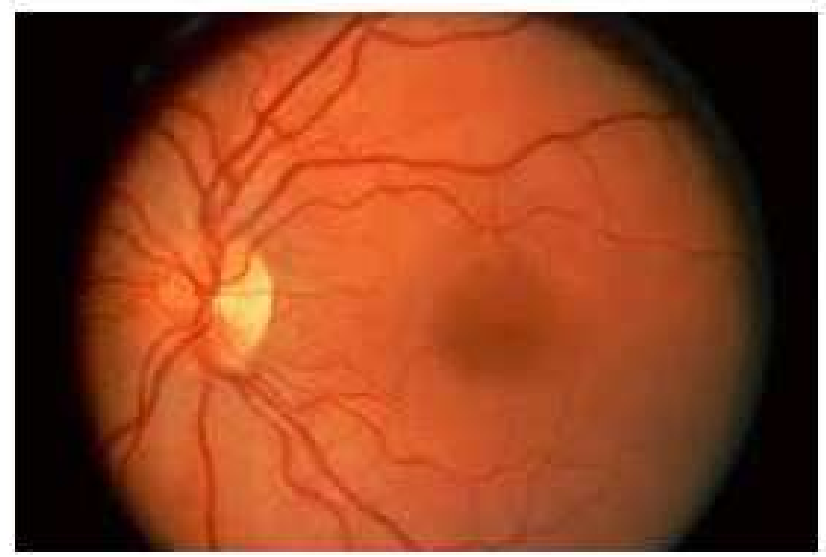

Figure 5: Bulls eye maculopathy following treatment.

\section{Discussion}

A diagnosis of SLE can be made based on a constellation of clinical features, ancillary investigations and laboratory tests. The disease has a course marked by remissions and exacerbations and assessment of ophthalmic problems may be indicators for overall disease activity. The American College of Rheumatology criteria established in 1982[5], was used to make a diagnosis of SLE but did not include ophthalmic features as necessary to diagnose SLE. The Systemic Lupus International Collaborating Clinics (SLICC) [6] group gave a revised, validated and updated classification in 2012. The British Isles Lupus Assessment Group index of disease activity (BILAG 2004) [7] gave a new classification featuring ophthalmic problems as an important criterion. A knowledge of these various diagnostic criteria helps the clinician to recognize SLE and to further divide this complex multisystem disease based on the target organ [8].

The patient seeks the consultation of the general practitioner for an eye problem and rheumatologist or physician if there is associated systemic disease. It is crucial at these early stages that symptoms are recognized and patients are referred to the ophthalmologist to prevent blindness that can occur in progressive disease. In our patient population we observed that ocular pain, congestion and inflammation is indicative of adnexal or anterior segment disease and symptoms such as blurring of vision or visual field defects indicate posterior segment or neuro ophthalmic disease.

Ophthalmic features can be varied and the spectrum of involvement can be adnexal, orbital, anterior segment, posterior segment or neuroophthalmic in manifestation [9]. Corneal signs of SLE are mostly seen as ocular surface epitheliopathy secondary to KCS or peripheral keratitis. The incidence of epicleritis as the presenting sign was high in our patients. It depicts active systemic disease and is usually less responsive to non- steroidal anti- inflammatory medications unlike in idiopathic episcleritis and scleritis. Reduction of signs and complete resolution was noted only after control and improvement in systemic status of SLE. Posterior segment involvement was less common in our study but was more symptomatic with a potential for vision loss. In our patient population, the commonest ocular manifestations were KCS, vasculitis and episcleritis. These ocular signs were reliable predictors of presence and activity of SLE. Vasculitis was seen in the early stages of SLE and was confirmed on fundus fluorescein angiography. The detection of retinal vasculitis should prompt a search for systemic vasculitis and both require aggressive immunosuppressive therapy [10]. Some of the retinal signs in SLE can occur secondary to hypertension which it is difficult to distinguish if due to immune complex deposition in SLE or due to hypertension. However, systemic treatment with corticosteroids and immunosuppressive drugs reduces the ocular features dramatically [11]. In our patient, local ophthalmic treatment was done using topical and periocular corticosteroid injections, lubricant eye drops for KCS and antibiotic ointment for peripheral corneal ulcers. In patients with capillary non perfusion and retinal ischemia, panretinal photocoagulation was done after confirming the extent of ischemia with fundus fluorescein angiography. Systemic management was done using tablet prednisolone $1 \mathrm{mg} / \mathrm{kg}$ / body weight, azathioprine $150 \mathrm{mg}$ daily and tapered to $50 \mathrm{mg}$, 
hydroxychloroquine $300-400 \mathrm{mg}$ daily. These drugs were given in combination or alone depending on the severity and stage of the disease. However when using immunosuppressive treatment, the patient should be monitored at regular intervals because of the risk of development of infections. A case of cytomegalovirus retinitis in a patient with SLE has been reported [12] and the risk of other infections is high during treatment.

Optic disc pallor that we saw in some of our patients was because of secondary optic atrophy which occurred on resolution of optic disc edema. Presence of superficial retinal hemorrhages, cotton-wool spots and disc edema may or may not be reflections of a concomitant hypertension in SLE. They can represent an independent effect of the inflammation and damage in ocular tissues, and they occur in the absence of hypertension [13]. The optic neuropathy in SLE is commonly bilateral and patient presents with acute painless loss of vision. Typical altitudinal or accurate field defects on perimetry can be seen in the presence or absence of optic disc swelling. The edema and defects are believed to occur because of occlusion of small vessels of the optic nerves with demyelination and axonal necrosis in severe cases [14]. Lupus induced involvement of choroid [15], eye lids [16], orbit [17] and ocular ischemia [18] have been described in the past but we did not encounter any such sign in our patients.

The appearance and resolution of retinopathy could be correlated with exacerbations and remissions of the systemic condition. Also, studies have shown that there is a decreased survival rate in patients with SLE retinopathy than in those without SLE associated retinopathy [19], [20].

Optic neuropathy can result in irreversible visual loss and recurrences cause further deterioration. Coordinated treatment strategies are important for reducing ocular morbidity and complications leading to blindness. Hence eye pain and visual impairment during any stage of the disease should be referred immediately as it requires urgent assessment and treatment by the ophthalmologist. Scleritis and retinopathy are absolute indications for immunosuppression while other manifestations are responsive to local eye drops and oral NSAIDS or steroid treatment. Drugs used in the treatment of SLE can themselves cause ocular problems [21]. Before starting treatment in SLE, ophthalmic evaluation and blood investigations need to be performed.

\section{Conclusion}

Detection of ocular lesions and in specific retinopathy is therefore of crucial importance, both visually and for prognosis of systemic disease. For those on treatment, during each review, systemic prognosis, slit lamp examination, ophthalmoscopy, color vision, visual acuity and visual fields tests have to be performed and monitored by an ophthalmologist. Early suspicion and recognition by the rheumatologist and prompt intervention by the ophthalmologist will prevent visual loss and improve prognosis in these patients.

Funding: Nil, Conflict of interest: None initiated. Permission from IRB: Yes

\section{References}

1. Preble JM, Silpa-archa S, Foster CS. Ocular involvement in systemic lupus erythematosus. CurrOpinOphthalmol. 2015(Nov); 26(6): 540-5. doi: 10.1097/ICU.0000000000000209.

2. Silpa-archa S, Lee JJ, Foster CS. Ocular manifestations in systemic lupus erythematosus. British Journal of Ophthalmology. 2016 Jan; 100(1):135-41. Doi: 10.1136/bjophthalmol- 2015- 306629. Epub 2015 Apr 22.

3. Tikly M, Navarra S.V. Lupus in the developing world - is it any different? Best PractRes Clin Rheumatol. 2008 Aug; 229(4): 643-55. Doi: 10.1016/j.berh. 2008.05.003.

4. D’Cruz D.P, Khamashta M.A, Hughes G.R. Systemic lupus erythematosus. Lancet.2007 Feb 17; 369(9561):587-596.

5. Tan EM, Cohen AS, Fries JF. The 1982 revised criteria for the classification of systemic lupus erythematosus. Arthritis Rheum. 1982 Nov;25(11): 1271-7.

6. Yu C, Gershwin ME, Chang C. Diagnostic criteria for systemic lupus erythematosus: a critical review. J Autoimmun. 2014 Feb- Mar; 48-49:10-3.doi: 10.1016/j.jaut.2014.01.004. Epub2014 Jan 21.

7. Isenberg DA, Rahman A, Allen E. BILAG 2004. Development and initial validation of an updated version of the British Isles Lupus Assessment Group's disease activity index for patients with systemic lupus 
erythematosus. Rheumatology(Oxford).2005 Jul; 44(7): 902-6.Epub 2005 Apr 6.

8. Karpik AG, Schwartz MM, Dickey LE, Streeten BW, Roberts JL. Ocular immune reactants inpatients dying with systemic lupus erythematosus. Clin Immunol Immunopathol. 1985 June; 35(3): 295-312.

9. Arevalo JF, Lowder CY, Muci- Mendoza R. Ocular manifestations of systemic lupus erythematosus. CurrOpinOphthalmol. 2002 (Dec); 13(6): 404-10.

10. Gold D.H, Morris A, Henkind P. Ocular findings in systemic lupus erythematosus. Br J Ophthalmol. 1972 Nov; 56(11):800- 4.

11. Davies JB, Rao PK. Ocular manifestations of systemic lupus erythematosus. Curr Opin Ophthalmol. 2008 Nov; 19(6): 512-8.

12. Kelkar A, Kelkar J, Kelkar S, Bhirud S, Biswas J. Cytomegalovirus retinitis in a seronegative patient with systemic lupus erythematosus on immunosuppressive therapy .J Ophthalmic Inflamm Infect. 2011 Sep: 1(3): 129-32. Doi 10. 1007/s12348-010-0017-3. Epub 2011 Apr 3.

13. Nguyen QD, Foster CS. Systemic lupus erythematosus and the eye. Int Ophthalmol Clin. 1998 Winter; 38 (1): 33-60.

14. Read RW. Clinical mini-review: systemic lupus erythematosus and the eye. Ocul Immunol Inflamm. 2004 June; 12(2): 87-99.
15. Laviña AM, Agarwal A, Hunyor A, Gass JD. Lupus choroidopathy and choroidal effusions. Retina 2002 Oct;22(5):643-47.

16. Huey C, Jakobiec FA, Iwamoto $T$, Kennedy R, Farmer ER, Green WR. Discoid lupus erythematosus of the eyelids. Ophthalmology.1983 Dec; 90 (12): 138998.

17. Norden D, Weinberg J, Schumacher HR, Keenan G, Freundlich B. Bilateral periorbital oedema in systemic lupus erythematosus. J Immunol.1993;20: 2158- 60.

18. Stavrou P, Murray PI, Batta K, Gordon C. Acute ocular ischemia and orbital inflammation associated with systemic lupus erythematosus. $\mathrm{Br} \mathrm{J}$ Ophthalmol. 2002 Apr; 86(4): 474-5.

19. Peponis V, KyttarisVC,Tyradellis C, Vergados I, Sitaras NM. Ocular manifestations of systemic lupus erythematosus: a clinical review. Lupus .2006; 15:3-12.

20. Coppeto J, Lessell S. Retinopathy in systemic lupus erythematosus. Arch Ophthalmol. 1997 May; 95(5): 794-7.

21. Foster CS. Immunosuppressive therapy for external ocular inflammatory disease. Ophthalmology. 1980 Feb: 87(2): 140-50.

\section{How to cite this article?}

Sruthi MS, Annamalai R, Muthukumar M.An analysis of ocular features and vision loss at presentation and after treatment in systemic lupus erythematosus in 60 patients. Int J Med Res Rev 2016;4(5):741-746.doi: 10.17511/ijmrr.2016.i05.13. 